\title{
Effect of introduction of colposcopy into a district general hospital
}

\author{
C. F. PILL* \\ M.R.C.O.G., F.R.A.C.O.G.
}

\author{
A. T. LETCHWORTH \\ M.D., F.R.C.O.G.
}

\author{
A. D. NOBLE \\ F.R.C.S., F.R.C.O.G.
}

Royal Hampshire County Hospital, Winchester, Hants SO22 5DG

\begin{abstract}
Summary
Management of cervical intra-epithelial neoplasia, in a district general hospital, was examined for two 22month periods before and after the introduction of colposcopy. This technique enables the clinician to evaluate the extent and severity of pre-malignant change, and makes treatment, using less radical destructive techniques, possible. The method of conservative treatment in this hospital was diathermy, and the cure rate of cases so treated was 97\%. The use of diathermy treatment resulted in a $75 \%$ reduction in the incidence of cone biopsy in women below the age of $\mathbf{4 0}$ years.

A further advantage of colposcopy has been recognized, which is that some women with "mild or moderate changes" are discovered to have more severe lesions, and receive appropriate treatment without delay. It follows that all patients with an abnormal smear should be referred for colposcopy, unless there is an obvious local cause of the abnormality, such as easily treatable infection, and that the post-treatment smear reverts to normal.

In this hospital the cost of establishing a colposcopic service was low, and the cost benefits, as well as the improved management of cervical intraepithelial neoplasia suggests that the service should be introduced in all district general hospitals.
\end{abstract}

KEY WORDS: cervical carcinoma, cervical intraepithelial neoplasia, diathermy, hysterectomy.

\section{Introduction}

Colposcopy is now established as a valuable way of evaluating inflammatory, benign, pre-malignant, and early malignant lesions of the cervix, but gynaecologists in the United Kingdom have tended to lag behind their colleagues in the rest of Europe, Australia, and the United States of America, and are only now beginning to introduce this service. Colposcopy is recommended for the patient whose cervical smear test is abnormal, to enable accurate assessment of possible pre-malignant change. It has been generally agreed that premalignant change in the cervix should be described as cervical intra-epithelial neoplasia (CIN) (Richard, 1966); CIN I is equivalent to the previous description of mild dysplasia; CIN II is equivalent to moderate dysplasia; CIN III is equivalent to severe dysplasia/carcinoma in situ.

This study examines the financial and medical implications of introducing colposcopy to a district general hospital.

\section{Patients and methods}

Patients from the Winchester Health District (total population approximately 200,000 ) with abnormal smears were seen in the Gynaecology Unit at the Royal Hampshire County Hospital. Patients were referred from their general practitioners or family planning clinic, where smears had been taken, either routinely or as part of the investigation of the patient's presenting symptoms.

Colposcopy was introduced in June 1979 and from that time all patients with abnormal smears rwere referred for colposcopic examination. In this study we evaluate the management of patients whose cervical smear test is abnormal before and after the introduction of colposcopy. The control period was the 22 months before colposcopy was introduced, and the study period was the first 22 months of colposcopic assessment.

In the control period, 66 patients with histologically proven CIN III were treated, and during this period the only available treatment of CIN III was either cone biopsy or hysterectomy (Fig. 1). Cone biopsy was the treatment of choice, especially for women whose families were not complete, but hysterectomy was advised if the cone biopsy suggested incomplete removal of the pre-malignant area, if the patient had other disabling gynaecological symptoms, or wished to be sterilized.

In the study period, 121 patients with histologically proven CIN III were treated. During this period all patients referred with abnormal smears were examined with the colposcope. This permitted the extent and severity of any dysplastic lesions to be assessed, 
Abnormal smeor
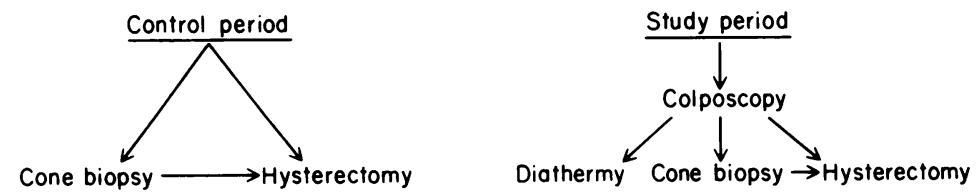

FIG. 1. Flow chart of procedures after abnormal smear found during control and study periods.

following preparation of the cervix with $3 \%$ acetic acid. Punch biopsies were then taken from the most abnormal areas, and examined histologically. In those cases where CIN III was evident histologically, and the lesion was completely visible, the treatment of choice was destruction by radical needle and ball diathermy (Chanen and Hollyhock, 1971). This was performed under general anaesthesia and preceded by a cervical dilatation and cervical and uterine curettage. The diathermy needle was inserted to a depth of $1 \mathrm{~cm}$, to ensure destruction of premalignant tissue, which may have involved cervical crypts (Anderson and Hartley, 1980), and the superficial tissue between the needle punctures was destroyed with ball diathermy. All patients were given a broad spectrum antibiotic and local antiseptic cream. The age of the patients treated in this manner ranged from 18 to 49 years. The patients were considered to be cured if on subsequent colposcopic examination at 4 months, 6 months, 12 months and 24 months no CIN lesion could be detected, and repeat cervical smears were negative.

\section{Costs}

The overall cost of equipment to establish the colposcopy clinic was $£ 2,004$, (colposcope $=£ 1,886$, endocervical speculum $=\mathfrak{£} 30$, cervical punch biopsy forceps $=£ 88$ ). The other equipment required was already available within the hospital. The patients were examined by lying on an ordinary couch fitted with lithotomy poles. Two out-patient sessions per week were required, and this was made available by the re-organisation of established clinics.

\section{Results}

During the control period, before the introduction of colposcopy, 66 patients with CIN III were treated. Of these $46(70 \%)$ had cone biopsies and $20(30 \%)$ underwent hysterectomy. Of the 121 patients in the study period, $32(26 \%)$ had cone biopsies, and 15 cases (13\%) underwent hysterectomy. The remaining 74 patients $(61 \%)$, were treated by needle and ball diathermy to the cervix, with 71 (96\%) being cured. In the three cases with persistent dysplasia further diathermy resulted in cure. In the group treated by diathermy there were no complications requiring readmission, and no patient was in hospital longer than $24 \mathrm{hr}$.

\section{Discussion}

While cone biopsy is an accepted method of treating CIN III, it carries the immediate problem of haemorrhage, and the long-term problems of midtrimester abortion and pre-term labour in subsequent pregnancies (Jones et al., 1976).

Colposcopic examination of all patients with abnormal smears allows an accurate assessment of the extent and nature of the CIN changes and facilitates accurate biopsy. As a result there is better diagnostic accuracy and the possibility of local treatment for selected patients. For those patients who do require a cone biopsy, colposcopy, by accurately delineating the lesion, enables a more limited procedure to be performed.

In this study a $75 \%$ reduction in the incidence of cone biopsy, in women below the age of 40 years, was obtained after the introduction of the colposcope. However, cone biopsies were necessary in $25 \%$ of the study group as the whole of the abnormal area could not be visualised, and it was therefore impossible to be certain that all the abnormal area would be destroyed by diathermy.

The study group contained 55 patients more than the control group, and there were a number of reasons for this. There was a $20 \%$ increase in the smears assessed by the cytological department during the study period, indicating an increased awareness of the problem of cervical dysplasia by our general practitioners. Following the introduction of colposcopy, patients with CIN I-II smears were referred to the hospital, whereas previous practice had been for follow-up cytology by the general practitioner. Of patients in the study group referred in this way with CIN I-II smears $17 \%$ had histologically proven CIN III lesions. Also the incidence of CIN III lesions in the community is increasing (Draper and Cook, 1983). The study group and control group were in other respects comparable.

The present study was undertaken in order to identify the changes in the pattern of work as well as the costs and benefits of introducing a colposcopy service to a district general hospital. In addition to the advantages already discussed, colposcopic examination leads to a more rational policy for treating patients with CIN I-II lesions. Colposcopy and biopsy enables a more accurate assessment to be 
made of the abnormal cervical lesion. As noted above $17 \%$ of patients with histological evidence of CIN III had smears suggesting CIN I-II. Other workers have also found that cytological smears are not reliable in predicting specific underlying lesions of the cervix. Hollyock and Fortune (1971) found a 40\% error between the cytological prediction and the subsequent histological findings. Before the introduction of colposcopy these patients would have remained untreated. While it is debatable whether formal treatment is required for CIN I-II lesions, the necessity for repeated smears causes considerable anxiety to many patients. During the study period 96 cases of CIN I-II lesions were treated with diathermy. A cone biopsy was performed for one case with CIN II, as the upper limit of the lesion could not be defined with the colposcopy.

- The cost of introducing a colposcopy service involved a modest capital outlay. There have also been revenue consequences as a result of the increase in the number of smears sent to the cytological department. To offset these increases, there has been a considerable saving in in-patient bed-days available, since the average stay in hospital for cervical diathermy is significantly shorter than that for cone biopsy.

There is a considerable and enlarging pool of gynaecologists, who have been trained in the technique of colposcopy, and it should not be difficult for most district general hospitals to establish this service. Over a period of two years in this Unit, one Australian-trained registrar instructed and trained two consultants, who are now able to continue the service.

We believe that colposcopy is a useful technique, which is not difficult to learn, and that most district general hospitals could introduce this service with both cost and clinical advantage.

\section{Acknowledgments}

We wish to thank Dr Sworn and Dr Vincenti (Pathologists), Mr Hawthorne (Chief Cytology Technician) and Miss Lee, without whose help this work would not have been possible.

\section{References}

Anderson, M.C. \& Hartley, R.B. (1980) Cervical crypt involvement by intra epithelial neoplasia. Obstetrics and Gynaecology, 55, 546.

Chanen, W. \& Hollyock, V.E. (1971) Colposcopy and electrocoagulation diathermy for cervical dysplasia and carcinoma-insitu. Obstetrics and Gynecology, 37, (4) 623.

DRAPER, G.J. \& COOK, G.A. (1983) Changing patterns of cervical cancer rates. (Editorial) British Medical Journal, 287, 510.

HOLLYOCK, V.E. \& FORTUNE, D.W. (1971) Cytological and histological correlation in $\mathbf{3 0 0}$ cases of cervical cone biopsy. Australian and New Zealand Journal of Obstetrics and Gynaecology, 11, 13.

Jones, J.M., SWEetNAM, P. \& HibBaRd, B.M. (1979) The outcome of pregnancy after cone biopsy of the cervix: a case-control study. British Journal of Obstetrics and Gynecology, 86, 913.

RICHARD, R.M. (1966) Natural history of cervical intra-epithelial neoplasia. Clinical Obstetrics and Gynaecology, 86, 703.

STAFL, A. \& Wilkinson, E.J. (1979) Cervical and vaginal intraepithelial neoplasia. In: Recent Advances in Obstetrics and Gynaecology (Eds. J. Stallworthy and G. Bourne), p. 155 Churchill Livingstone, London, p.155.

(Accepted 23 November 1983) 